\title{
Effects of Probiotics, Prebiotics and Synbiotic Supplementation on Cognitive Impairment: A Review
}

\section{Murugan Mukilan ${ }^{1,2^{*}}$}

\author{
${ }^{1}$ Advanced Technology Development Centre, Indian Institute of Technology, Kharagpur 721 302, West Bengal, India \\ ${ }^{2}$ Assistant Professor, Department of Biotechnology, Sri Ramakrishna College of Arts \& Science (SRCAS), Coimbatore 641 006, Tamil Nadu, India
}

Received - December 22, 2021; Revision - February 15, 2021; Accepted - February 25, 2022

Available Online - February 28, 2022

DOI: http://dx.doi.org/10.18006/2022.10(1).1.11

KEYWORDS
Learning
Memory
Probiotics
Lactobacillus
Bifidobacterium
Alzheimer's disease

\begin{abstract}
Learning and memory formation are the two essential terms widely used in the field of cognition. Learning can be defined as acquiring new information or skills. Memory is formed due to changes in the neuronal system as a result of continuous stimulus exposure. Both learning and memory are fundamental processes that occur in all living organisms. Memory is broadly categorized into two different categories such as short-term memory (STM) and long-term memory (LTM). Compared to STM, LTM plays an essential role in the day-to-day activities of different living organisms. LTM requires RNA and protein synthesis-dependent mechanisms for memory storage, which lasts up to their lifetime. LTM formation is initiated when the neurotransmitters are released from the presynaptic neuron; further released neurotransmitters bind with their respective receptors present in the postsynaptic neuron and initiate the calcium influx. Calcium influx results in the further activation of molecules involved in the neuronal signaling pathway and results in memory formation. Present review reports the outcome of recent studies which showed that probiotic supplement is responsible for the retrieval of memory in case of memory impairment and its uses in the treatment of neurodegenerative disorders like mild cognitive impairment (MCI), Alzheimer's disease (AD). Recent research studies were shown that probiotic microorganisms may positively regulate neurotransmitter release and increase the calcium influx, brain derived neurotrophic factor (BDNF), and N-methyl-D-aspartate receptor (NMDAR) and plays a pivotal role in the LTM formation in gut-dysbiosed \& memory-impaired animal models.
\end{abstract}

* Corresponding author

E-mail: mukilan@srcas.ac.in (Murugan Mukilan)

Peer review under responsibility of Journal of Experimental Biology and Agricultural Sciences.

Production and Hosting by Horizon Publisher India [HPI] (http://www.horizonpublisherindia.in/).

All rights reserved.
All the articles published by Journal of Experimental Biology and Agricultural Sciences are licensed under a Creative Commons Attribution-NonCommercial 4.0 International License Based on a work at www.jebas.org.

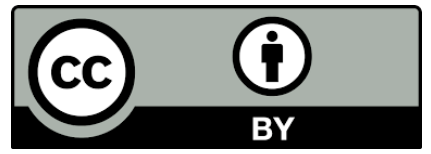




\section{Introduction}

Synaptic plasticity refers to the activity-dependent changes that occur in synapses and plays a prominent role in memory formation (Kind and Neumann 2000; Sweatt 2001; Abraham et al. 2019). Several experimental animal models were shown that changes in the synaptic strength are occurred due to repeated stimulus exposure/activity. These changes are referred to as long-term potentiation (LTP) (Malenka et al. 1992; Thiels and Klann 2001; Abraham et al. 2019; Evans et al. 2021). LTP was first identified by terjelømoin the year 1966. After the discovery of LTP, several studies have been attempted to focus on the change of locus, pre/postsynaptic currents. Recently, it is accepted that postsynaptic signaling mechanisms are most needed for the LTP induction and serve as a primary mechanism to maintain increased synaptic response (Waltereit et al. 2001; Yuste and Bonhoeffer 2001; Evans et al. 2021).

At the initial phase of memory processing in the brain, memory formation may be inhibited by various hindrances like seizures, trauma, inactivation of neuronal pathways, brain lesions, and inactivation of specific transcription factors, translation, or specific blockade of molecular pathways. During the initial memory processing, memory impairment happened due to various reasons such as trauma, seizures, neuronal pathways inactivation/blockade, of inhibition of neuronal transcription factors. Thereby transcription plays an essential role in memory consolidation or its retainment. Long-term plasticity changes result in long-term facilitation (LTF), LTP, and long-term depression (LTD) which requires both transcription and protein synthesis (Baker-Herman and Mitchell 2002; Roberts and Glanzman 2003; Evans et al. 2021 Lin et al. 2021). Several animal studies have been shown that longterm memory (LTM) needed de novo protein synthesis during the first few hours of training (Schafe and LeDoux 2000; Scharf et al. 2002; Igaz et al. 2006; Abraham and Williams 2007; Lin et al. 2021; Evans et al. 2021).

The learning process is associated with activity-dependent changes and results in neurotransmitters' release (Lovinger 2010; Bai and Suzuki 2020). Neurotransmitter release results in the activation of neuronal signaling pathway with the help of several proteins like protein kinase A (PKA), extracellular signal-regulated kinase-1/2 (ERK-1/2), mitogen-activated protein kinase (MAPK), and cyclic AMP response element-binding protein-1 (CREB-1) (Yoon and Seger 2006; Ganesh et al. 2010; Ganesh et al. 2012; Mukilan et al. 2015; García-Pardo et al. 2016; Mukilan et al. 2018a, 2018b). Activation of ERK-1/2 results in the phosphorylation of CREB-1 (Peng et al. 2010). Further, phosphorylated CREB-1 results in the activation of the immediate early gene (IEG) cascade and other postsynaptic density protein (PSD), which results in the formation of LTM (Ganesh et al. 2010; Ganesh et al. 2012; Mukilan et al. 2015; Mukilan et al. 2018a, 2018b).
In normal healthy persons, gut microbiota was needed for the proper functioning of the central nervous system (CNS) through endocrine, neural, and immune pathways (Grenham et al. 2011; Moloney et al. 2014; Ma et al. 2019). Pathogenic infection or stress exposure stimulates the secretion of corticotrophin-releasing factors (CRF) via the hypothalamus-pituitary-adrenal axis from the brain. CRF disturbs the gut microbiota and increases the production of endotoxins (Bailey and Coe1999; Mayer et al. 2015; Misak et al. 2020). These endotoxins limit the secretion of serotonin and catecholamines (Linthorst and Reul 1998; Fung et al. 2017; Yang and Chiu 2017). Serotonin and catecholamines increase interaction between the gut microbiota and CNS. By this signaling pathway, CNS maintains homeostasis. Impairment or dysregulation of this signaling pathway resulted in autism, Alzheimer's disease, memory impairment, and neurodegenerative disorders (Linthorst and Reul 1998; Fung et al. 2017; Salami 2021). Recent studies were shown that treatment with probiotic microorganisms was used to overcome the impaired cognitive decline in autism and Alzheimer's disease (Petrof et al. 2013; Choi and Choi 2016; Asl et al. 2019; Morshedi et al. 2020).

Probiotics are beneficial living microorganisms, when it was taken in an appropriate dose it supports the host in many ways like the improvement of cognition, immune system, and also it will supply needed antioxidants (FAO/WHO 2002; Sherman et al. 2009; Kwok et al. 2014). Compared to probiotics, prebiotics was formed by the fermentation of non-digestible ingredients by the beneficial gut microbiota. These prebiotics enhance the growth and activity of beneficial gut microorganisms. The formed probiotic/prebiotic precursor molecules increase the growth and metabolic activity of beneficial microorganisms present in the gut. Thereby it increased the short-chain fatty acid (SCFA) level and also modifies alphasynuclein protein (Franco-Robles and López 2015; MarkowiakKopeć and Ślizewska 2020). SCFA were used for the regulation of neurotransmitter release and have a direct effect on the expression level of brain-derived neurotrophic factor (BDNF) and nerve growth factor (NGF). It will also be shown that SCFA act as an essential molecule for the signaling between the gut microbiota and brain (Montarolo et al. 1986; Dale et al. 1987; Gräff and Tsai 2013; Buffington et al. 2014; Franco-Robles and López 2015; Heyck and Ibarra 2019; Markowiak-Kopeć and Ślizewska 2020).

Recent research reports have shown that the gut microbiota plays an important role in the regulation of gut-brain axis (Wong et al. 2018; Misak et al. 2020). Some gut bacterial strains may also regulate the level of neurotransmitters like $\gamma$-aminobutyric acid (GABA)(Wong et al. 2003). Recent studies have shown that various neurotransmitter levels were regulated by some bacterial strains present in the gut. Regulated neurotransmitters may include $\gamma$-aminobutyric acid (GABA) (Wong et al. 2003; O'Donnell et al. 2020), serotonin (5-HT), dopamine (DA), and noradrenaline (NA) 
(Chen et al. 2017). These neurotransmitters potentially regulate calcium influx and other molecules involved in the formation of LTM (Romo-Araiza et al. 2018; Rezaeiasl et al. 2019; Yang et al. 2020). Present review article trying to explore the role of prebiotics and probiotics in the formation of LTM and its uses in the treatment of memory impairment in neurogenerative disorders like Alzheimer's disease (AD), epilepsy, Parkinson's disease (PD), autism spectrum disorders (ASD).

\section{Effect of neuro inflammation on memory impairment}

Degeneration of neurons/neuroinflammation within the CNS are associated with the decline in memory formation (Romo-Araiza et al. 2018). Mild cognitive impairment (MCI) is regarded as the intermittent stage of memory decline between healthy apoptosis and dementia (Tajiri et al. 2017; Romo-Araiza et al. 2018; Tobin et al 2019). MCI results in the loss of neurons in different brain regions like the hippocampus. Loss of neurons may also be related to the defects of mitochondria and oxidative stress. Oxidative stress results in increased levels of proinflammatory cytokines, which results in neuroinflammation. In later stages, there is a probability of conversion of MCI to Alzheimer's disease (Baierle et al. 2015; Zhao et al. 2015; Romo-Araiza et al. 2018; Tobin et al. 2019).
Neuroinflammation is generally associated with cognitive memory decline. This neuroinflammation results in the inflammation of the hippocampal tissue region. Hippocampal tissue inflammation will result in decreased plasticity changes, which results in impaired LTM formation (Di Filippo et al. 2013; Zhao et al. 2019). Decreased plasticity changes result in the low-level synthesis of glutamate and downregulation of $\mathrm{N}$-methyl-D-asparate receptor (NMDAR) in the hippocampus's CA1 and CA3 region (Kumar and Mehta 2011; Bye and McDonald 2019).The downregulation of NMDAR results in impaired LTM formation (Rosi et al. 2005; Di Filippo et al. 2013; Baierle et al. 2015). Other than NMDAR, Brain-Derived Neurotrophic Factor (BDNF) is also downregulated during neuroinflammation/gut dysbiosis which results in impaired memory formation (Ryan and Nolan 2016; Mora 2013).

\section{Role of short-chain fatty acid (SCFA) on memory} enhancement

Intestinal probiotic microorganisms are needed for short-chain fatty acid (SCFA) production. This SCFA are made up of carboxylic acids, these carboxylic acid are present with the aliphatic tails of 1-6 carbon. In these 6 carbon molecules, 3 carbon molecules $\{$ acetate $(\mathrm{C} 2)$, propionate $(\mathrm{C} 3)$, and butyrate $(\mathrm{C} 4)\}$ were

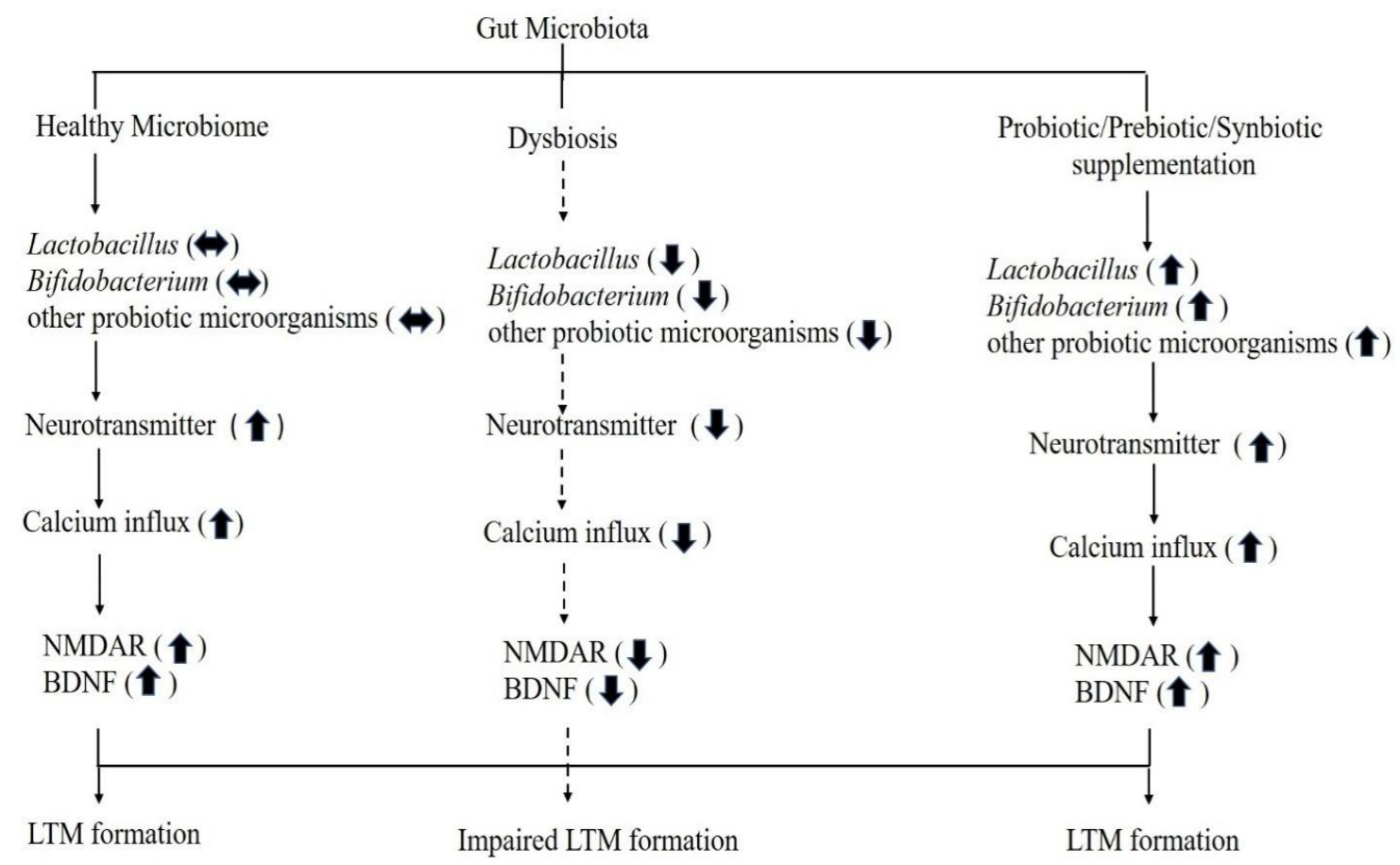

Figure 1 Effect of gut microbiota in the long-term memory (LTM) formation. A) Gut microbiota plays a main role in the development of LTM by the release of neurotransmitters (ex. serotonin) in its normal state and further results in the increase of calcium influx and brain derived neurotrophic factor (BDNF) and N-methyl-D-aspartate receptor (NMDAR). B) dysbiosis affects the normal level of gut microbiota, low level of gut microbiota inhibits the neurotransmitter release, decrease the calcium influx and downregulates BDNF \&NMDAR.Low levels of BDNF \& NMDAR results in the impaired LTM formation C) Treatment with probiotics restores the normal gut microbiota and restores the LTM memory formation. 
produced by anaerobic fermentation of dietary fibers (DF) by the intestinal microorganisms. In these aliphatic tails, three different carbon molecules will be synthesized from the dietary fibers with the help of gut microbiota by anaerobic digestion. Some of the carbon molecules were produced by the Acetate are the most abundant SCFA produced in the gut. Acetate was made from acetyl-CoA from glycolysis (den Besten et al. 2013; Duncan et al. 2002). Propionate and butyrate formation occurs from the carbohydrate metabolism of glycolysis (Louis and Flint 2017). Levels of SCFA decline with age and microbial imbalance. Microbial imbalance results in the increase of pathogenic bacteria Proteobacterium and results in the brain's inflammation (Caracciolo et al. 2014; Romo-Araiza et al. 2018)

Brüssow stated that both probiotics and prebiotics were used for the production of SCFA, increasing neurotrophic factors and neuronal plasticity. Agave inulin a most commonly used prebiotic stimulates the growth of Enterococcus faecium (probiotic bacterium), thereby indirectly promoting butyrate synthesis (Huang et al. 2017). Among the three SCFA, butyrate functions as a histone deacetylase inhibitor, enhancing the upregulation of BDNF expression in the different regions of the brain and inhibiting the secretion of proinflammatory cytokines (Park et al. 2016; Kim et al. 2014). Recent research findings were shown that healthy gut microbiota increases butyrate production; the presence of enough butyrate level reduces neuroinflammation and increases BDNF, NMDAR levels in the specific brain region, and upregulation of BDNF, NMDAR results in increased synaptic plasticity (Stilling et al. 2016; Pineda-Rodriguez et al. 2017; Canani et al. 2018).

\section{Effect of probiotics on long term memory formation}

Several intestinal microbial species influence the physiology, development, and maintenance of an individual's health in the form of gut microbiota. Gut microbiota can be differentiated into three major categories viz., bacteria, viruses, and fungi. These microorganisms regulate intestinal $\mathrm{pH}$ and act as a preventive barrier against infectious agents. A healthy level/ equilibrium of intestinal microbiota plays an important role in the maintenance of proper health. A healthy state shows the mutual relationship between gut microbiota and the nervous system (Jiang et al. 2017). Equilibrium of intestinal microbiota was mainly affected by different lifestyles like diet (Gentile and Weir 2018), alcohol consumption (Hillemacher et al. 2018), smoking (Savin et al. 2018), and changes in circadian rhythm (Kaczmarek et al. 2017). However, alteration in the gut microbiota may induce changes in brain activity and also cause neurological disorders like $\mathrm{AD}$ (Angelucci et al. 2019). Affected intestinal microbiota equilibrium comes back to normal by consuming probiotics/prebiotics/ synbiotic supplementation (Kaczmarek et al. 2017; Gentile and
Weir 2018; Hillemacher et al. 2018; Savin et al. 2018; Hadizadeh et al. 2019)

Probiotics produce beneficial effects on the host's health (Mukherjee et al. 2018). Most bacteria are used as probiotics; probiotic bacteria are needed to synthesize substances required for a host. The presence of a sustainable number of necessary substances prevents inflammation and related diseases (Mukherjee et al. 2018). The most commonly used probiotic strains include Lactobacillus and Bifidobacteria. Both of these strains are present in yogurt, fermented cheese, and vegetables. Other than probiotics, we also consumed dietary fibers; anaerobic fermentation of dietary fibres results in the formation of SCFA (Daliri et al. 2018; Mukherjee et al. 2018). This type of probiotic microorganisms may synthesize and release different neurotransmitters like serotonin, GABA, histamine, and dopamine (Gareau 2014; Dinan and Cryan 2017). Several gut microorganisms are responsible for the synthesis of neurotransmitters like dopamine, noradrenaline, serotonin, GABA, acetylcholine, and histamine which plays a main role in long-term memory formation (Stanaszek et al. 1977; Tsavkelova et al. 2000; Landete et al. 2007; Shishov et al. 2009; Özoğul et al. 2012; Pokusaeva et al. 2017). However, gut microbiota imbalances play an important role in the deficient synthesis of biogenic amines and neurotransmitters which will have a direct impact on long-term memory formation (Matsumoto et al. 2013). Prebiotic or probiotic treatment increases neurotransmitter level and improves the cognitive functions in memory-impaired animal models (Lyte 2011; Barrett 2012; Dinan 2015; Sarkar et al. 2016; Bermúdez-Humarán et al. 2019).

Neurotransmitters produced by the probiotic microorganisms at first cross the blood-brain barrier and reach the central nervous system (CNS) (Pokusaeva et al. 2017). Among the pool of neurotransmitters, serotonin (5-HT) is synthesized from the amino acid tryptophan. At first, amino acid tryptophan is converted to 5hydroxytrptophan (5-HTP) with the help of tryptophan hydroxylase. Formed 5-HTP is again reconverted to 5-HT by the aromatic amino acid decarboxylase (AACD) (Adell et al. 2002). This 5-HT is first reported to be involved in learning and memory formation by the 1980s (Altman and Normile 1988). This 5 -HT is having multiple neural markers like receptors and transporters (McCorvy and Roth 2015). Further, 5-HT is synthesized from serotonin synthesizing neurons. Serotonin synthesizing neurons are present along the brainstem's midline; the most prominently present in raphe nuclei. Axons of these serotonin synthesizing neurons innervate almost all regions of the brain. Activation of 5HT receptors stimulates adenyl cyclase (AC), this AC induces the rapid increase of cyclic adenosine monophosphate (cAMP) levels which directly stimulates protein kinase A (PKA) activity. Further, PKA increases MAPK/ERK kinase (Mohamed et al. 2005). ERK $1 / 2$ cascade activation is necessary to consolidate the learning 
Probiotics

Bifidobacterium bifidum

B. lactis

B. infantis

B. Longum

Lactobacillus fermentum

L. casei

L. plantarum

L. acidophilus
Prebiotics

Fructo oligosaccharides (FOS)

Galacto-oligosaccharides (GOS)

Bimuno-galacto-oligosaccharides (B-GOS)

Non-carbohydrate oligosaccharides

Fructans

Oligosaccharide carbohydrates

Inulin enriched FOS

Mixture of FOS and GOS

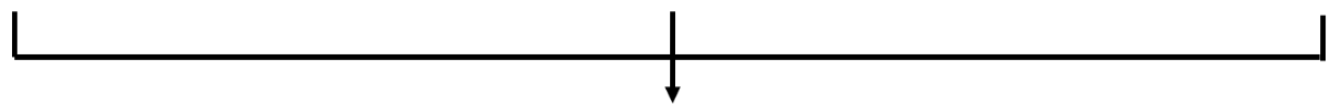

\section{Modulation of Gut microbiota \\ Short Chain Fatty Acids (SCFAs) production Synthesis of neurotransmitter precursor molecules $\rightarrow \begin{gathered}\text { Improvement of } \\ \text { gut-brain axis }\end{gathered}$ Neurotransmitter regulation Regulation of HPA axis}

Figure 2 Role of probiotics/prebiotics/synbiotics on the improvement of gut-brain axis

paradigm (Ganesh et al. 2010; Ganesh et al. 2012; Mukilan et al. 2015; Mukilan et al. 2018a; Mukilan et al. 2018b). Compared to the ERK cascade, BDNF is also involved in long-term memory formation. BDNF is needed for stimulating hippocampalneocortical interaction during the consolidation of memory (Bambah-Mukku et al. 2014). During the consolidation of longterm memory, hippocampal-neocortical interactions were stimulated by the BDNF (Bambah-Mukku et al. 2014; Miranda et al. 2019).

5 Effect of prebiotic and synbiotic supplementation on memory formation

Recent studies were evidenced that early life intake of prebiotic is associated with improvement of cognitive abilities, LTM formation in the early and middle stages of life. During early life, gut microbial colonization is needed for the development of the neuronal structure and its function (Williams et al. 2016). Treatment of neonatal rats with a galacto-oligosaccharide prebiotic (BGOS) increases the expression of N-methyl-D-asparate receptor (NMDAR) subunit-GluN2A, synaptic proteins, and brain-derivedneurotrophic factor (BDNF) in the hippocampal brain region and also alters the neurotransmission during memory impairment. Other than BGOS, oral administration of 2'-fucosyllactose (2'-FL) also showed a higher level of long-term potentiation (LTP) (Williams et al. 2016; Oliveros et al. 2016). Other than prebiotic, synbiotic (probiotic and prebiotic) supplementation is also used for the development of memory in memory-impaired animal models.
Romo-Araiza et al. (2018) stated the effect of probiotic and prebiotic supplementation on spatial memory formation.

6 Impact of probiotic supplementation on neural dysfunctions

Recent research findings showed that probiotic supplementation plays an essential role in the prevention of neural dysfunctions by regulating age-related cognitive impairment. Song et al. (2013) tested the effect of prebiotic galactooligosaccharides (GOS) on the neuroprotective effect in amyotrophic lateral sclerosis (ALS). The outcome of the study showed that the administration of GOS reduced motor neuron loss, improved the consequences of atrophy, and also stated the role of probiotic yogurt in the disease onset improvement. Other than GOS, probiotic yogurt administration also delayed disease onset and increased the lifespan of treated mice. Yang et al. (2020) investigated the effect of probiotics on the deficits of intestinal gut microbiota and restores cognitive function using the probiotic preparation of Bifidobacterium lactis, B. bifidum, Lactobacillus casei, and L. acidophilus (ProBiotic-4) on SAMP8 (Senescence-accelerated mouse prone 8) mice. Oral administration of ProBiotic-4 for 4, 8, and 12 weeks significantly improves the deficits of the microbiota-gut-brain axis and cognitive function (Yang et al. 2020).

\section{Conclusion}

Recent studies were shown that probiotic, prebiotic, and synbiotic supplementation is used as a clinical tool to treat memory decline 
in $\mathrm{AD}$, mild cognitive impairment (MCI), and type 2 diabetes mellitus (T2DM) induced memory impairment. The most commonly anti-inflammatory property of the synbiotic supplementation is used for the treatment of neurological disorders and cognition decline in MCI. Compared to MCI, T2DM is a common metabolic disease that leads to memory dysfunction in the brain and is also associated with complications of gut-brain disorders. Some of the important research findings were shown the impact of probiotic, prebiotic, and symbiotic supplementation on the improvement of cognitive complications and plays the main role in the strengthening of neurotransmitter concentration (especially serotonin), BDNF/TrkB/CREB signaling pathways in different brain regions. Strengthening of this neuronal signaling pathway results in the improvement of memory during cognitive decline. Along with this supplementation, SCFAs will also play a positive role on the brain-gut axis. These SCFAs may also be produced by anaerobic fermentation of non-digestible ingredients with the help of certain probiotic microorganisms. The newly formed SCFAs are also involved in the new protein synthesis, enhance histone acetylation and do long-term plasticity changes in the brain. The long-term plasticity changes were enhanced by histone acetylation and could be improved with the help of HDAC inhibitors (HDACi). These SCFAs also act against neuroinflammation and inhibits the expression of proinflammatory cytokines in the presence of butyrate and sodium butyrate. Simply, SCFA increases the levels of BDNF, NMDAR through the supplementation of pre and probiotics along with food. Thereby SCFA plays an essential role in reducing the risk of Alzheimer's disease development due to aging and brain injuries. Other than synbiotic and SCFAs, ProBiotic-4 is also used to treat gut microbiota dysbiosis, which will result in the improvement of cognitive function. However considering the data reviewed here, shown that probiotic, prebiotic, and synbiotic supplementation intake reduces cognitive impairment, memory decline and restores synaptic plasticity and specific gut microbiota changes occur due to lead-led memory impairment, MCI, AD, and neurodegenerative disorders. Other than the restoration of gut microbiota, these supplementations are also used for the restoration of memory impairment and morphological abnormalities of dendritic spines. Thereby present review focused on the effect of probiotic, prebiotic, and synbiotic supplementation on the treatment of memory impairment caused due to aging, MCI, and the role gutbrain axis in the long-term memory formation. This review elaborates the role of gut microbiota on the synthesis of the neurotransmitter, activation of neuronal molecules in long-term memory formation.

\section{Acknowledgments}

MM was supported by the Institutional Post-Doctoral Fellowship of the Indian Institute of Technology, Kharagpur, India. This work is also supported by the SEED MONEY grant of Sri Ramakrishna College of Arts \& Science (SRCAS), Coimbatore.

\section{Funding}

This research received no external funding.

\section{Ethical approval}

This article does not contain any studies with human participants or animals performed by the author.

\section{Conflicts of Interest}

The author declare no conflict of interest.

\section{References}

Abraham W.C., Jones, O.D., \& Glanzman, D.L. (2019). Is plasticity of synapses the mechanism of long-term memory storage? Npj Science of learning, 4, 9.

Abraham, W.C., \& Williams, J.M. (2007). LTP maintenance and its protein synthesis-dependence. Neurobiology of Learning and Memory, 89,260-268.

Adell, A., Celada, P., Abellán, M.T., \& Artigas, F. (2002). Origin and functional role of the extracellular serotonin in the midbrain raphe nuclei. Brain Research Reviews, 39, 154-180.

Altman, H.J., \& Normile, H.J. (1988). What is the nature of the role of the serotonergic nervous system in learning and memory: prospects for development of an effective treatment strategy for senile dementia. Neurobiology of Aging, 9, 627-638.

Angelucci, F., Cehova, K., Amlerova, J., \& Hort, J. (2019). Antibiotics, gut microbiota, and alzheimer's disease. Journal of Neuroinflammation, 16, 108.

Asl, Z.R., Sepehri, G., \& Salami, M. (2019). Probiotic treatment improves the impaired spatial cognitive performance and restores synaptic plasticity in an animal model of alzheimer's disease. Behavioural Brain Research, 376, 112183.

Bai Y., \& Suzuki, T. (2020). Activity-Dependent Synaptic Plasticity in Drosophla melanogaster. Frontiers in physiology, 11, 161.

Baierle, M., Nascimento, S.N., Moro, A.M., Brucker, et al. (2015). Relationship between inflammation and oxidative stress and cognitive decline in the institutionalized elderly. Oxidative Medicine and Cellular Longevity, 2015, 804198.

Bailey, M.T, \& Coe, C.L. (1999). Maternal separation disrupts the integrity of the intestinal microflora in infant rhesus monkeys. Developmental Psychobiology, 35, 146-155. 
Baker-Herman, T.L., \& Mitchell, G.S. (2002). Phrenic long-term facilitation requires spinal serotonin receptor activation and protein synthesis. Journal of Neuroscience, 22, 6239-6246.

Bambah-Mukku, D., Travaglia, A., Chen, D.Y., Pollonini, G., \& Alberini, C.M. (2014). A positive autoregulatory BDNF feedback loop via $\mathrm{C} / \mathrm{EBP} 3$ mediates hippocampal memory consolidation. Journal of Neuroscience, 34, 12547-12559.

Barrett, E. (2012). $\gamma$ - Aminobutyric acid production by culturable bacteria from the human intestine. Journal of Applied Microbiology, 113, 411-417.

Bermúdez-Humarán, L.G., Salinas, E., Ortiz, G.G., RamirezJirano, L.J., Morales, J.A., \& Bitzer-Quintero, O.K. (2019). From Probiotics to Psychobiotics: Live Beneficial Bacteria Which Act on the Brain-Gut Axis. Nutrients, 11, 890.

Buffington, S.A., Huang, W., \& Costa-Mattioli, M. (2014). Translational control in synaptic plasticity and cognitive dysfunction. Annual Review of Neuroscience, 37, 17-38.

Bye, C.M., \& McDonald, R.J. (2019). A Specific Role of Hippocamal NMDA Receptors and Arc Protein in Rapid Encoding of Novel Environmental Representations and a More General Long-Term Consolidation function. Frontiers in Behavioral Neuroscience, 13, 8.

Canani, R.B., Filippis, F.D., Nocerino, R., \& Paparo, L. (2018). Gut microbiota composition and butyrate production in children affected by non-IgE-mediated cow's milk allergy. Scientific Reports, 8, 12500.

Caracciolo, B., Xu, W., Collin, S., \& Fratiglioni, L. (2014). Cognitive decline, dietary factors and gut-brain interactions Mechanisms of Ageing and Development, 136-137,59-69.

Chen, D., Yang, X., Yang, J., Lai, G., et al. (2017). Prebiotic effect of fructooligosaccharides from Morinda officinalis on alzheimer's disease in rodent models by targeting the microbiota-gut-brain axis. Frontiers in Aging Neuroscience, 9, 403.

Choi, H.H., \& Choi, Y.S. (2016). Fecal microbiota transplantation: current applications, effectiveness and future perspectives. Clinical Endoscopy, 49, 257-265.

Dale, N., Kandel, E.R., \& Schacher, S. (1987). Serotonin produces long-term changes in the excitability of aplysia sensory neurons in cuture that depend on new protein synthesis. Journal of Neuroscience, 7, 2232-8.

Daliri, E.B., Tango, C.N., Lee, B.H., \& Oh, D. (2018). Human microbiome restoration and safety. International Journal of Medical Microbiology, 308, 487-497. den Besten, G., Eunen, K.V., Groen, A.K., Venema, K., Reijngoud, D.J., \& Bakker B.M. (2013). The role of short-chain fatty acids in the interplay between diet, gut microbiota, and host energy metabolism. Journal of Lipid Research, 54, 2325-2340.

Di Filippo, M., Chiasserini, D., Gardoni, F., Viviani, B., et al. (2013). Effects of central and peripheral inflammation on hippocampal synaptic plasticity. Neurobiology of Disease, 52, 229-236.

Dinan, T.G. (2015). Collective unconscious: how gut microbes shape human behavior. Journal of Psychiatric Research, 63, 1-9.

Dinan, T.G., \& Cryan, J.F. (2017). The Microbiome-Gut-Brain Axis in Health and Disease. Gastroenterology Clinics of North America, 46, 77-89.

Duncan, S.H., Barcenilla, A., Stewart, C.S., Pryde, S.E., \& Flint, H.J. (2002). Acetate utilization and butyeyl coenzyme A (CoA): acetate-CoA transferase in butyrate-producing bacteria from the human large intestine. Applied and Environmental Microbiology, 68, 5186-90.

Evans, H.T., Blackmore, D., Götz, J., \& Bodea, L. (2021). De novo proteomic methods for examining the molecular mechanisms underpinning long-term memory. Brain Research Bulletin, 169, 94-103.

Franco-Robles, E., \& López, M. (2015). Implication of fructans in health: Immunomodulatory and antioxidant mechanisms. Scientific World Journal, 2015, 289267.

Fung, T.C., Olson, C.A., \& Hsiao, E.Y. (2017). Interactions between the microbiota, immune and nervous systems in health and disease. Nature Neuroscience, 20, 145-155.

Ganesh, A., Bogdanowicz, W., Balamurugan, K., Varman, D.R., \& Rajan, K.E. (2012). Egr-1antisense oligodeoxynucleotide administration into the olfactory bulb impairs olfactory learning in the greater short-nosed fruit bat Cynopterus sphinx. Brain Research, 1471, 33-45.

Ganesh, A., Bogdanowicz, W., Haupt, M, Marimuthu, G., \& Rajan, K.E. (2010). Role of olfactory bulb serotonin in olfactory learning in the greater short-nosed fruit bat, Cynopterus sphinx (Chiroptera: Pteropodidae). Brain Research, 1352, 108-117.

García-Pardo, M.P., Roger-Sanchez, C., Rodríguez-Arias, M., Miñarro, J., \& Aguilar, M.A. (2016). Pharmacological modulation of protein kinases as a new approach to treat addiction to cocaine and opiates. European Journal of Pharmacology, 781, 10-24.

Gareau, M.G. (2014). Microbiota-gut-brain axis and cognitive function. Advances in Experimental Medicine and Biology, 817, 357-371. 
Gentile, C.L., \& Weir, T.L. (2018). The gut microbiota at the intersection of diet and human health. Science, 362, 776-780.

Gräff, J., \& Tsai, L.H. (2013). Histone acetylation: molecular mnemonics on the chromatin. Nature Reveiws Neuroscience, 14 97-111.

Grenham, S., Clarke, G., Cryan, J.F., \& Dinan, T.G. (2011). Braingut-microbe communication in health and disease. Frontiers in Physiology, 2, 94.

Hadizadeh, M., Hamidi, G.A., \& Salami, M. (2019). Probiotic supplementation improves the cognitive function and the anxietylike behaviors in the stressed rats. Iranian Journal of Basic Medical Sciences, 22, 506-514.

Heyck, M., \& Ibarra, A. (2019). Microbiota and memory: a symbiotic therapy to counter cognitive decline? Brain Circulation, $5,124-129$.

Hillemacher, T., Bachmann, O., Kahl, K.G., \& Frieling, H. (2018) Alcohol, microbiome, and their effect on psychiatric disorders. Progress in Neuropsychopharmacology and Biological Psychiatry, $85,105-115$

Huang, W., Guo, H., Deng, X., Zhu, T., et al. (2017). Short-chain fatty acids inhibit oxdative stress and inflammation in mesangial cells induced by high glucose and lipopolysaccharide. Experimental and Clinical Endocrinology and Diabetes, 125, 98105.

Igaz, L.M., Winograd, M., Cammarota, M., Izquierdo, L.A., et al. (2006). Early activation of extracellular signal-regulated kinase signaling pathway in the hippocampus is required for short-term memory formation of a fear-motivated learning. Cellular and Molecular Neurobiology, 2006, 989-1002.

Jiang, C., Li, G., Huang, P., Liu, Z., \& Zhao, B. (2017). The gut microbiota and alzheimer's disease. Journal of Alzheimer's Disease, 58, 1-15.

FAO/WHO. (2002). working group report on drafting the guidelines for the evaluation of probiotics in food. London, Ontario, Canada, April 30 and May 1, 2002.

Kaczmarek, J.L., Thompson, S.V., \& Holscher, H.D. (2017). Complex interactions of circadian rhythms, eating behaviors, and the gastrointestinal microbiota and their potential impact on health. Nutrition Reviews, 75, 673-682.

Kim, C.H., Park, J., \& Kim, M. (2014). Gut microbiota-derived short-chain fatty acids. $\mathrm{T}$ cells and inflammation. Immune Network, 14, 277-288.
Kind, P.C., \& Neumann, P.E. (2001). Plasticity: downstream of glutamate. Trends in Neuroscience, 24, 553-555.

Kumar, A., \& Mehta, M.R. (2011). Frequency-Dependent Changes in NMDAR-Dependent Synaptic plasticity. Frontiers in Computational Neuroscience, 5, 38.

Kwok, L.Y., Wang, L., Zhang, J., Guo, Z., \& Zhang, H. (2014). A pilot study on the effect of Lactobacillus casei Zhang on intestinal microbiota parameters in chinese subjects of different ages. Beneficial Microbes, 5, 2955-304.

Landete, J.M., Ferrer, S., \& Pardo, I. (2007). Biogenic amine production by lactic acid bacteria, acetic bacteria and yeast isolated from wine. Food Control, 18,1569-1574.

Lin, H., Chen, C., de Belle, J.S., Tully, T., \& Chiang, A. (2021). CREBA and CREBB in two identified neurons gate long-term memory formation in Drosophila. Proceedings of the National Academy of Sciencesof the United States of America, 118, e2100624118.

Linthorst, A.C., \& Reul, J.M. (1998). Brain neurotransmission during peripheral inflammation. Annals of the New York Academy of Sciences, 840, 139-152.

Louis, P., \& Flint, H.J. (2017). Formation of propionate and butyrate by the human colonic microbiota. Environmental Microbiology, 19, 29-41.

Lovinger, D. M. (2010). Neurotransmitter roles in synaptic modulation, plasticity and learning in the dorsal striatum. Neuropharmacology, 58, 951-961.

Lyte, M. (2011). Probiotics function mechanistically as delivery vehicles for neuroactive compounds: microbial endocrinology in the design and use of probiotics. Bioessays, 33, 574-581.

Ma, Q., Xing, C., Long, W., Wang, H.Y., Liu, Q., \& Wang, R. (2019). Impact of microbiota on central nervous system and neurological diseases: the gut-brain axis. Journal of Neuroinflammation, 16, 53.

Malenka, R.C., Lancaster, B., \& Zucker, R.S. (1992). Temporal limits on the rise in postsynaptic calcium required for the induction of long-term potentiation. Neuron, 9, 121-128.

Markowiak-Kopeć, P., \& Ślizewska, K. (2020). The Effect of Probiotics on the Production of Short-Chain Fatty Acids by Human Intetinal Microbiome. Nutrients 12, 1107.

Matsumoto, M., Kibe, R.K., Ooga, T., Aiba, Y., et al. (2013). Cerebral low-molecular metabolites influenced by intestinal microbiota: a pilot study. Frontiers in System Neuroscience, 7,9. 
Mayer, E.A., Tillisch, K., \& Gupta, A. (2015). Gutbrain axis and the microbiota. Journal of Clinical Investigation, 125, 926-938.

McCorvy, J.D., \& Roth, B.L. (2015). Structure and function of serotonin G protein-coupled receptors. Pharmacology and Therapeutics, 150, 129-142.

Miranda, M., Morici, J.F., Zanoni, M.B., \& Bekinschtein, P. (2019). Brain-Derived Neurotrophic Factor: A Key Molecule for memory in the Healthy and the Pathological Brain. Frontiers in Cellular Neuroscience, 13, 363.

Misiak, B., Łoniewski, I., Marlicz, W., Freydeca, W., et al. (2020). The HPA axis dysregulation in severe mental illness: can we shift the blame to gut microbiota. Progress in NeuroPsychopharmacology and Biological Psychiatry, 102, 109951.

Mohamed, H.A., Yao, W., Fioravante, D., Smolen, P., \& Byrne, J.H. (2005). Camp-response elements in Aplysia creb1, creb2 and Ap-uch promoters: implications for feedback loops modulating long term memory. Journal of Biological Chemistry, 280, 27035 27043.

Moloney, R.D., Desbonnet, L., Clarke, G., Dinan, T.G., \& Cryan, J.F. (2014). The microbiome: stress, health and disease. Mammalian Genome, 25, 49-74.

Montarolo, P.G., Goelet, P., Castellucci, V.F., Morgan, J., Kandel, E.R., \& Schacher, S. (1986). A Critical period for macromolecular synthesis in long-term heterosynaptic facilitation in Aplysia. Science, 234, 1249-1254.

Mora, F. (2013). Successful brain aging: plasticity, environmental enrichment and lifestyle. Dialogues in Clinical Neuroscience, 15, 45-52.

Morshedi, M., Saghafi-Asl, M., \& Hosseinifard, E.S. (2020). The potential therapeutic effects of the gut microbiome manipulation by symbiotic containing-Lactobacillus plantarum on neuropsychological performance of diabetic rats. Journal of Translational Medicine, 18, 18.

Mukherjee, S., Joardar, N., Sengupta, S., \& Babu, S.P.S. (2018). Gut microbes as future therapeutics in treating inflammatory and infectious diseases: lessons from recent findings. The Journal of Nutritional Biochemistry, 61, 111-128.

Mukilan, M., Bogdanowicz, W., Marimuthu, G., \& Rajan, K.E. (2018a).Odour discrimination learning in the Indian greater shortnosed fruit bat (Cynopterus sphinx): differential expression of Egr1, C-fos and PP-1 in the olfactory bulb, amygdala and hippocampus. Journal of Experimental Biology, 221, jeb175364.
Mukilan, M., Rajathei, D.M., Jeyaraj, E., Kayalvizhi, N., \& Rajan, K.E. (2018b). MiR-132 regulated olfactory bulb proteins linked to olfactory learning in greater short-nosed fruit bat Cynopterus sphinx. Gene, 671, 10-20.

Mukilan, M., Varman, D.R., Sudhakar, S., \& Rajan, K.E. (2015). Activity-dependent expression of miR-132 regulates immediate early gene induction during olfactory learning in the greater shortnosed fruit bat, Cynopterus sphinx. Neurobiology of Learning and Memory, 120, 41-51.

O’Donnell, M.P., Fox, B.W., Chao, P., Schroeder, F.C., \& Sengupta, P. (2020). A neurotransmitter produced by gut bacteria modulates host sensory behaviour. Nature, 583, 415-420.

Oliveros, E., Ramirez, M., Vazquez, E., Barranco, A., et al. (2016). Oral supplementation of 2'-fucosyllactose during lactation improves memory and learning in rats. The Journal of Nutritional Biochemistry, 31, 20-27.

Özoğul, F.K.E., özoğul, Y., \& Özoğul, I. (2012). The function of lactic acid bacteria on biogenic amines production by food-borne pathogens in arginine decarboxylase broth. Food Science and Technology Research, 18,795-804.

Park, J., Goergen, C.J., HogenEsch, H., \& Kim, C.H. (2016). Chronically elevated levels of short-chain fatty acids induce T cellmediated ureteristis and hydronephrosis. The Journal of Immunology, 196, 2388-400.

Peng, S., Zhang, Y., Zhang, J., Wang, H., \& Ren, B. (2010). ERK in learning and memory: a review of recent research. International Journal of Molecular Sciences, 11,222-232.

Petrof, E.O., Claud, E.C., Gloor, G.B., \& Allen-Vercoe, E. (2013). Microbial ecosystems therapeutics: a new paradigm in medicine? Beneficial Microbes, 4, 53-65.

Pineda-Rodriguez, B., Toscano-Tejeida, D., Garcia-Vences, E., Rodriquez-Barrera, R., et al. (2017). Anterior chamber associated immune deviation used as a neuroprotective strategy in rats with spinal cord injury. PLos One, 12, e0188506.

Pokusaeva, K., Johnson, C., Luk, B., \& Uribe, G. (2017). GABAproducing Bifidobacterium dentium modulates visceral sensitivity in the intestine. Neurogastroenterology \& Motility, 29, e12904.

Rezaeiasl, Z., Salami, M., \& Sepehri, G. (2019). The effects of probiotic Lactobacillus and Bifidobacterium strains on memory and learning behavior, long-term potentiation (LTP), and some biochemical parameters in B-amyloid-induced rat's model of alzheimer's disease. Preventive Nutrition and Food Science, 24, 265-273. 
Roberts, A.C., Glanzman, D.L. (2003). Learning in aplysia: looking at synaptic plasticity from both sides. Trends in Neuroscience, 26, 662-670.

Romo-Araiza, A., Gutiérrez-salmeán, G., Galván, E.J., HernándezFrausto, M., et al. (2018). Probiotics and prebiotics as a therapeutic strategy to improve memory in a model of middle-aged rats Frontiers in Aging Neuroscience, 10, 416.

Rosi, S., Ramirez-Amaya, V., Vazdarjanova, A., Worley, P.F., Barnes, C.A., \& Wenk G.L. (2005). Neuroinflammation alters the hippocampal pattern of behaviorally induced Arc expression. Journal of Neuroscience, 25, 723-731.

Ryan, S.M., \& Nolan, Y.M. (2016). Neuroinflammation negatively affects adult hippocampal neurogenesis and cognition: can exercise compensate? Neuroscience \& Biobehavioral Reviews, 61,121-131.

Salami, M (2021). Interplay of good bacteria and Central Nervous System: Cognitive Aspects and Mechanistic Considerations. Frontiers in Neuroscience, 15, 613120.

Sarkar, A., Lehto, S.M., Harty, S., Dinan, T.G., Cryan, J.F., \& Burnet, P.W.J. (2016). Psychobiotics and the manipulation of bacteria-gut-brain signals. Trends in Neuroscience, 39, 763781.

Savin, Z., Kivity, S., Yonath, H., \& Yehuda, S. (2018). Smoking and the intestinal microbiome. Archives of Microbiology, 200, 677-684.

Schafe, G.E., \& LeDoux, J.E. (2000). Memory consolidation of auditory pavlovian fear conditioning requires protein synthesis and protein kinase A in the amygdala. Journal of Neuroscience, 20, RC96.

Scharf, M.T., Woo, N.H., Lattal, K.M., Young, J.Z., Nguyen, P.V., \& Abel, T. (2002). Protein synthesis is required for the enhancement of long-term potentiation and long-term memory by spaced training. Journal of Neurophysiology, 87, 2770-2777.

Sherman, P.M., Ossa, J.C., \& Johnson-Henry, K. (2009). Unraveling mechanisms of action of probiotics. Nutrition in Clinical Practice, 24, 10-14.

Shishov, V.A.K.T., Kudrin, V.S., Oleskin, A.V. (2009). Amine neuromediators, their precursors, and oxidation products in the culture of Escherichia coli K-12. Prikl Biokhim Microbiol, 45, 550-554.

Song, L., Gao, Y., Zhang, X., \& Le, W. (2013). Galactooligosaccharide improves the animal survival and alleviates motor neuron death in SOD1G93A mose model of amyotrophic lateral sclerosis. Neuroscience, 246,281-90.
Stanaszek, P.M., Snell, J.F., \& O’Neill, J.J. (1977). Isolation, extraction, and measurement of acetylcholine from Lactobacillus plantarum. Applied and Environmental Microbiology, 34, 237-239.

Stilling, R.M., de Wouw, M.V., Clarke, G., Stanton, C., et al. (2016). The neuropharmacology of butyrate: The bread and butter of the microbiota-gut-brain axis? Neurochemistry International, 99, 110-132.

Sweatt, J.D. (2001). The neuronal MAP kinase cascade: A biochemical signal integration systemsubserving synaptic plasticity and memory. Journal of Neurochemistry, 76, 1-10.

Tajiri, N., Quach, D.M., Kaneko, Y., Wu, S., et al. (2017). NSI-19, a small molecule with neurogenic properties, exerts behavioral, and neurostructural benefits in stroke rats. Journal of Cellular Physiology, 232, 2731-2740.

Thiels, E., \& Klann, E. (2001). Extracellular signal-regulated kinase, synaptic plasticity and memory. Reviews in Neuroscience, 12, 327-345.

Tobin, M.K., Musaraca, K., Disouky, A., Shetti, A., et al. (2019). Human hippocampal neurogenesis persists in aged adults and alzheimer's disease patients. Cell Stem Cell, 24,974-982.

Tsavkelova, E.A., Botvinko, I.V., Kudrin, V.S., \& Oleskin, A.V. (2000). Detection of neurotransmitter amines in microorganisms with the use of high-performance liquid chromatography. Doklady Biochemistry and Biophysics, 372, 115-117.

Waltereit, R., Dammermann, B., Wulff, P., Scafidi, J., et al. (2001). Arg3.1/Arc mRNA induction by $\mathrm{Ca}^{2+}$ and AMP requires protein kinase A and mitogen-activated protein kinase/extracellular regulated kinase activation. Journal of Neuroscience, 21, 54845493.

Williams, S., Chen, L., Savignac, H., Tzortzis, G., Anthony, D.C., \& Burnet, P.W.J. (2016). Neonatal prebiotic (BGOS) supplementation increases the levels of synaptophysin, GluN2Asubunits and BDNF proteins in the adult rat hippocampus. Synapse, 70, 121-4.

Wong, C.B., Kobayashi, Y., \& Xiao, J. (2018). Probiotics for preventing cognitive impairment in alzheimer's disease. In Evrensel, A., \& Ünsalver, B.O. (eds), Gut Microbiota, IntechOpen. DOI: $10.5772 /$ intechopen.79088.

Wong, C.G.T., Bottiglieri, T., \& Snead, I.I.I. OC. (2003). GABA, $\gamma$-hydroxybutyric acid and neurological disease. Annals of Neurology, S6, S3-S12. 
Yang, N.J., \& Chiu, I.M. (2017). Bacterial signaling to the nervous system through toxins and metabolites. Journal of Molecular Biology, 429, 587-605.

Yang, X., Yu, D., Xue, L., Li, H., et al. (2020). Probiotics modulate the microbiota-gut-brain axis and improve memory deficits in aged SAMP8 mice. Acta Pharmaceutica Sinicia B, $10,475-487$.

Yoon, S., \& Seger, R. (2006). The extracellular signal-regulated kinase: multiple substrates regulate diverse cellular functions. Growth Factors, 24, 21-44.
Yuste, R., \& Bonhoeffer, T. (2001). Morphological changes in dendritic spines associated with long-term synaptic plasticity. Annual Review of Neuroscience, 24,1071-1089.

Zhao, J., Wei, B., Xiao, S., Lan, X., et al. (2019). Neuroinflammation induced by lipopolysaccharide causes cognitive impairment in mice. Scientific Reports, 9, 5790.

Zhao, X., Yuan, L., Feng, L., Xi, Y., et al. (2015). Association of dietary intake and lifestyle pattern with mild cognitive impairment in the elderly. The Journal of Nutrition, Health \& Aging, 19, 164168 . 